\title{
Robotic Wrist Rehabilitation With High-density Electromyographic Biofeedback for Reducing Co- activation of Antagonist Muscles and Improved Motor Coordination: A Feasibility Study
}

Imre CIKAJLO ( $\square$ imre.cikajlo@ir-rs.si )

University rehabilitation institute https://orcid.org/0000-0002-8428-7621

Matjaž Zadravec

University Rehabilitation Institute

Zlatko Matjačić

University Rehabilitation Institute

Filip Urh

Univerza v Mariboru Fakulteta elektrotehniko racunalnistvo informatiko

Martin Šavc

Univerza v Mariboru Fakulteta elektrotehniko racunalnistvo informatiko

Jernej Kranjec

Univerza v Mariboru Fakulteta elektrotehniko racunalnistvo informatiko

Matjaž Divjak

Univerza v Mariboru Fakulteta elektrotehniko racunalnistvo informatiko

Aljaž Frančič

Univerza v Mariboru Fakulteta elektrotehniko racunalnistvo informatiko

Božidar Potočnik

Univerza v Mariboru Fakulteta elektrotehniko racunalnistvo informatiko

Aleš Holobar

Univerza v Mariboru Fakulteta elektrotehniko racunalnistvo informatiko

\section{Methodology}

Keywords: rehabilitation, biofeedback, upper extremities, robotics, electromyography

Posted Date: June 15th, 2020

DOl: https://doi.org/10.21203/rs.3.rs-34303/v1

License: (c) (i) This work is licensed under a Creative Commons Attribution 4.0 International License.

Read Full License 
Page $2 / 18$ 


\section{Abstract}

Objectives: Electromygraphic (EMG) biofeedback has been successfully applied in stroke survivors for a few decades. Recently high-density (HD) electrodes were introduced in research and diagnostic EMG. The application of such electrodes may increase the reliability and selectivity, but requires information reduction for real-time biofeedback. We tested whether the use of the proposed feedback is feasible in minimization of inappropriate co-activation of wrist flexors/extensors controlled by the wrist rehabilitation robot.

Methods: The EMG of wrist flexors and extensors muscles has been assessed by arrays of $5 \times 13$ electrodes, reduced to $1 \times 4$ information matrix and provided as real-time biofeedback during wrist movement controlled by the robot. The designed task required to keep the contribution of agonist muscles within predefined limits. A feasibility study with a single patient with stroke was conducted to examine force tracking and reduction of activity of antagonists wrist muscles in 4 weeks.

Results: Force tracking signal and cumulative forecast errors decreased in 4 trials. The RMS activity of antagonist muscles significantly decreased (Kruskal Wallis, $c^{2}=3.330 * 10^{-9}$ ) during wrist flexion in the $4^{\text {th }}$ week of trials. The participant was able to follow the reduced HDEMG biofeedback.

Conclusions: The outcomes show that the surface HDEMG biofeedback has a potential to decrease the co-activation of antagonist muscles, wrist flexion in particular. Additionally the combination of force tracking and robot assisted biofeedback training may recover selective hand function in stroke survivors who would less likely to develop contractures.

Trial registration: The study is not a clinical trial. While human subjects are involved, they do not participate in a full rehabilitation intervention, and no health outcomes are examined.

\section{Introduction}

A neuromuscular system consists of the neural-system and musculoskeletal system and inherently provides a feedback to the brain in humans. Stroke as a consequence of the blood supply cut off (ischemic stroke, $85 \%$ of cases) or bleeding in the brain (haemorrhagic stroke, $15 \%$ of cases) often causes a lack of information or confusing information from neuromuscular systems. Therefore stroke survivors suffer from sensorimotor impairments and most of them use various biofeedback during the rehabilitation process to regain the ability to improve motor control by learning to control their responses [1]. The biofeedback provides information on specific biomedical variable, that can be measured directly or indirectly. In the clinical settings several types of biofeedback are used: visual, audio feedback of electromyogram, position, velocity, force values, etc [2] and can be classified either in physiological or biomechanical categories [3]. Potential benefits of electromyographic (EMG) biofeedback has been demonstrated in hemiplegic patients, using the biofeedback during the exercise for hand [4],[5]. The researchers found the EMG biofeedback as a promising tool and the most widely used and reported method of biofeedback in rehabilitation [3]. But despite of large number of RCTs further work is required, 
recent technologies in particular [2]. One of the recent studies reported that EMG biofeedback therapy has proven more effective than conventional physiotherapy for hand extension/flexion. Additionally, the authors suggested that such technology might be essential in rehabilitation of elderly patients with stroke [6].

One of the major drawback of the existing EMG technologies has been a low quality signal, artifacts, sensitivity and electrode failures. Recent advancement in technologies and signal processing enabled automatic detection of low quality signals, interpolation and generating a high density EMG map [7]. A high-density surface EMG has been used as an efficient diagnostic tools [8], particularly in the development of novel motor unit detection algorithms [9]. The high-density EMG (HDEMG) contains a large amount of information and to the best of our knowledge it has not been used as a biofeedback yet, but rather as an input to the myoelectric prosthetic hand control [10]. Also various combinations of the EMG biofeedback and neurobiofeedback in hand rehabilitation have been used to improve the reliability of the feedback and even demonstrated effectiveness comparable to the conventional occupation therapy [11].

Using the EMG biofeedback to learn how to coordinate the antagonist muscle groups in patients with motor disorders apparently reaches back to the 1980s. The participants of the study [12] were able to reduce the amount of inappropriate co-activation of wrist flexors or extensors when tracking a visual target. Almost four decades later the authors reported on similar findings in persons with chronic stroke. The participants reduced the co-activation of arm muscles in isometric conditions using EMG biofeedback with 3 pairs of muscles in combination with computer games [13]. 32 moderately or severely impaired participants showed significant improvement of range of motion, muscle co-activation and a potential for reducing spasticity.

In the proposed feasibility study we tested the advantages of using HDEMG in combination with biofeedback for reduction of inappropriate co-activation of wrist flexors/extensors controlled by the wrist rehabilitation robot. The application of high-density electrode arrays and reduction of information to the level of real-time biofeedback is certainly a novelty in post stroke rehabilitation. The objective of the feasibility study was to identify whether the person after stroke is able to learn how to control the agonist muscles without co-contraction of the antagonist muscles during isolated wrist movement with the novel type of biofeedback.

\section{Materials And Methods}

\section{Equipment}

The feasibility study anticipated isolated wrist flexion and extension. The movements were therefore controlled by the Universal Haptic Device (UHD) robot [14]. The robot opposed the movement to exert muscle forces and also measured forces and wrist positions at a sampling rate of $200 \mathrm{~Hz}$ with 12-bits resolution (PCI-6023E, National Instruments Inc., USA). Two arrays of $5 \times 13$ surface high-density EMG 
(HDEMG) electrodes (diameter of $1 \mathrm{~mm}$, inter-electrode distance of $8 \mathrm{~mm}$, OT Bioelettronica, Italy) were attached to the flexors and extensor muscles of the dominant forearm (Fig. 1). Electrode columns were approximately perpendicular to the muscle fibers, and covered about three-quarters of the forearm circumference. The recorded HDEMG signals were amplified, band-pass filtered $(3 \mathrm{~dB}, 10-900 \mathrm{~Hz})$ and sampled at $2048 \mathrm{~Hz}$, with 12 bits` resolution (USB EMG 2 amplifier, OT Bioelettronica, Italy). For the offline analysis only the robotic system generated a trigger signal during the wrist movement to synchronize the force signals with the HDEMG signals at both amplifiers. Later on the recorded force, position and HDEMG signals were synchronized and re-sampled for the off line analysis. If any of the electrodes fails to provide the signal, the signal would be eliminated from the analysis and from the real-time feedback.

Two setups were prepared for the feasibility study; a force feedback displayed online as a graphical feedback (Fig. 1 right) and the surface HDEMG biofeedback. The later provided information on flexors/extensors muscles activities in specific regions. The cumulative contribution of the agonist muscles were presented by a blue vertical bar. However, the target line would move higher, if the cumulative activity of antagonist muscles increases. The vertical green bar presented the time of keeping the agonist active above the target threshold and after the $10 \mathrm{~s}$ provided a smiley (Fig. 1 left).

\section{Subjects}

A 66-year old male chronic post-stroke subject (height $175 \mathrm{~cm}$, weight $98 \mathrm{~kg}$, hemiplegia, right-side affected) participated in the feasibility study carried out in the controlled laboratory environment of the University Rehabilitation Institute, Republic of Slovenia - Soča.

The inclusion criteria for the participant were: first unilateral stroke, low to medium hand spasticity level (Ashworth scale < 3), cognitive ability to follow the instructions (MMSE > 24). Exclusion criteria: arthritis, swan neck, nerve disfunction and other neuromuscular disorders not a consequence of stroke.

The feasibility study was conducted in accordance with the Declaration of Helsinki, and was approved by the institutional committee for medical ethics. The participant received a detailed explanation of the study and provided written informed consent before the commencement of the study.

\section{Force Tracking}

The participant's arm and hand was fixed in the frame of the UHD that limited the movement to the wrist flexion and extension only (Fig. 1 right). The robot provided linear resistance defined by the spring-force mechanism [15] during the wrist movement. We estimated that the applied wrist force had been adequate to the UHD's resisting force also measured in all three dimensions.

We have developed a trapezoid shape trajectory for force tracking in the sagittal plane of the wrist's axis (Fy). The ramp slope of the signal was adequate to the maximal flexion/extension movement speed that 
the participant could achieve. The participant was seated, put the arm in the UHD frame and was asked to follow the time-course of the reference force signal displayed on the LCD monitor (Fig. 1 right) by:

- moving the wrist downward (wrist flexion force)

- moving the wrist upward (wrist extension force)

The trajectory was designed to require actions in the following order: starting from a position of force equilibrium, followed by a maximal wrist extension, returning to the equilibrium position with $2 \mathrm{~s}$ of rest, followed by a maximal wrist flexion. The actions were repeated 10 times, lasting approximately $150 \mathrm{~s}$ in total. The participant repeated the procedure 4 times with 10 minutes breaks.

The analysis comprised of computing the tracking signal (TS) error, the cumulative forecast error (CFE) and the mean absolute error (MAE). The CFE is calculated as the total sum of errors (e) - bias: differences between the reference signal and measured wrist force;

$$
C F E=\sum_{j=1}^{n} \mid e_{j}(t)
$$

TS was calculated as a quotient between the CFE and MAD. The MAD is the mean absolute deviation and measures the total error of the signal regardless of the sign.

Better compliance with the reference signal results in lower MAD and CFE values [16]. MAE is a common measure for accuracy of continuous signals and measures the absolute differences between reference (y) and human generated force $(y)$ :

$$
M A E=\frac{1}{n} \sum_{j=1}^{n}\left|y_{j}-\dot{y}_{j}\right|
$$

\section{Emg Biofeedback}

The setup for the EMG biofeedback was similar to the force tracking; participant's arm and hand was fixed in the frame of the UHD that limited the movement to the wrist flexion and extension only (Fig. 1 left). But the task design required to keep the force at the specified intensity level and activate only flexors muscle group at flexion or only extensor muscle group at extension. The activities of specific muscle groups were assessed with the HDEMG equipment. The surface HDEMG is highly interferential signal consisting of contribution from several motor units (MU). Thus it is difficult for accurate interpretation. The information on muscle activity depends on the distribution of motor units within the muscle tissue and increases variability of muscle excitation across different muscles. Therefore a method for efficient selection of MU was needed [17]. The major challenge was how to reduce the amount of HDEMG data and present it as a real-time biofeedback. Usually a single information is provided as a feedback [3], but 
we found that fusion of all rows and 4 columns of electrodes can efficiently eliminate overestimation of muscle activation [18]. The RMS (root mean square) of the signals, recorded by electrodes in columns 14 presented the first circle, columns 4-7 the second, 7-10 the third and the columns from 10-13 the last circle on the display (Fig. 2). A single column was common for the neighboring circles. The desired muscle activities (e.g. m.flexors at wrist flexion) were presented by blue color in circles of the upper row, while the circles of the bottom row became red at the undesired activity (e.g. m. extensors at wrist flexion). Thus the participant was able to follow the muscle activities in 8 circles (4 per row) of different color in addition to the cumulative muscle group activity on the target display. Since the wrist flexor or extensor muscles' activities were related to the same movement, the feedback information was compliant with the recommendations for biofeedback [19].

The goal of the task was to move the wrist in the specified direction (flexion/extension) and keep the cumulative agonist muscles activity within the target levels for $10 \mathrm{~s}$. The target levels varied according to the agonists vs antagonists cumulative activity ratio. The cumulative activity of antagonists moved the target threshold for agonists cumulative activity higher. A successful attempt was rewarded with a smiley and a small splashing animation on the display (Fig. 2). The initial target levels were set before the daily assessment by measuring three $3 \mathrm{~s}$ long maximum voluntary (MVC) contractions of tested muscles during robot controlled wrist flexion and extension.

The feasibility study anticipated three to four sessions with 10 repetitions of wrist flexions and extensions at $\sim 40-60 \% \mathrm{MVC}$ level in stroke survivals in a single day. The protocol lasted 4 consecutive weeks.

Following the feasibility study protocol the HDEMG signals were examined off-line one by one with computer fault detection algorithm. The algorithm was looking for excessive and zero amplitudes in each particular electrode. The zero amplitude signal was considered a faulty electrode and was removed from further computation. Similarly the excessive amplitudes as a consequence of movement artifacts were also removed [20].

The HDEMG signal of each electrode was rectified after the signal being detrended. Followed by filtering with the 2nd order Butterworth low-pass filter with a cut-off frequency of $20 \mathrm{~Hz}$ [20] forward and backward, the HDEMG data of the daily sessions were normalized by their maximal values. These values were obtained from the MVC recordings on that day (Fig. 2 bellow), eliminating the effect of the electrodes placement and yielding the excitation in \% of maximal excitation, making the recordings comparable across weeks.

The objective of the robot supported EMG feedback was to decrease the activity of antagonist muscles and perform the controlled wrist force generation with agonist muscle groups only, if possible. Thus, the RMS values of HDEMG for each repetition, session and week were calculated separately for flexors and extensor muscle groups for wrist flexion and wrist extension. The envelopes and spatially averaged RMS values were also compared between 1st and 4th week for both movement directions. We expected that the participant would be capable of following the EMG biofeedback and keeping the target within the 
range activating the agonists muscles only. In addition spatially averaged RMS values were statistically analyzed; normal distribution was rejected in all values, therefore Kruskal-Wallis test (unpaired comparison) was used to check the statistical differences of RMS values across weeks. A correction of significant effects was performed by a Bonferroni test with significance level $P<0.05$.

Matlab (MathWorks, Natick MA, USA) was used as a main tool for raw data extraction and filtering and Matlab Statistical Toolbox for statistical analysis. All missing data were interpolated and non-number signals of faulted electrodes were immediately eliminated.

\section{Results}

\section{Force tracking}

The participant demonstrated progress in tracking the reference force (Fig. 3). The CFE value has significantly decreased for both movement directions, particularly at the wrist flexion. Similar results were found in TS. However, both error indicators show that major adaptation took place in the first three trials. But the absolute difference (MAE) between the reference and provided wrist force shows that the performance of the participant could have been even better, if there was less delay. Presumably the participant's reaction time became longer (approx. $150 \mathrm{~ms}$ ) in the 4th trial.

\section{Hdemg Biofeedback}

The comparison of outcomes after 4 weeks demonstrated that the normalized and rectified HDEMG amplitudes of antagonist muscles decreased at wrist flexion, but were unexpectedly larger at wrist extension (Fig. 4). We have noticed clear activities of agonist muscles in the raw EMG (gray shaded) but also constant and weaker activation of antagonist muscles at wrist flexion in the 4th week. However, the co-contractions of agonist and antagonist muscle groups at wrist extension were almost simultaneous. Further analysis of RMS of EMG signal reveals that flexors acting as antagonists were even more active in the 4th week (Fig. 4 below).

Furthermore, the detailed analysis of RMS HDEMG for each single electrode (Fig. 5) shows the decreased activity of extensors, the antagonists muscles during wrist flexion - the intensity decreased in the 4th week around all electrodes except at column 10 between rows 2 and 4 . The activity of the antagonists muscles (flexors) at wrist extension demonstrated less intensity in the 2nd week, but the activity rose in the $3 \mathrm{rd}$ week in the middle column of the electrode array. The overall activity of the flexors at wrist extension was more intense in the 4th week.

In the Fig. 6 median values of the RMS HDEMG, interquartile range (IQR), 1.5 times IQR and 95\% confidence intervals are presented. There was a great variability for HDEMG activity in the 3rd week. The Kruskal-Wallis statistical check provided an outcome in favor of our hypothesis and confirmed the antagonists activity decreased in wrist flexion $\left(\chi^{2}=2.658^{*} 10^{-37}\right)$. Further comparison of weekly 
measurements shows that the true medians of the extensors (antagonists) at wrist flexion for the 1st week and the 4th week were different and there was a significant difference of means $\left(\chi^{2}=3.330 * 10^{-9}\right)$. The Kruskal-Wallis test was also significant for the flexors (antagonists) activity at wrist extension $\left(\chi^{2}=\right.$ 0.00047).

\section{Discussion}

\section{Force tracking}

The force tracking has been considered an important task for hand function in progressive neuromuscular diseases, particularly following changes in muscular weakness. Stroke survivors usually do not experience problems with their grip force control despite evident muscular weakness [21]. They more often produce large force tracking errors. In our study this was noticed in isolated wrist flexion / extension in the 4 th trial. The certain level of spasticity and fatigue have been common impairments following stroke and could also be considered a cause for the larger reaction time and thus larger tracking mean absolute error. The spasticity can be effectively reduced by a multidisciplinary approach, a combination of medication and physical therapy [22]. Therefore it is possible that wrist force control training temporarily decreased the level of spasticity as stroke survivors are less likely to develop contractures if they are provided physical therapy for hand function recovery [23]. Nevertheless, the participant with stroke learned to track the reference force through the training and trials. Based on the shown results of cumulative error and the TS we may anticipate that such approach is worth further consideration.

\section{Hdemg Biofeedback As A Rehabilitation Tool}

The outcomes of the proposed approach with HDEMG suggest that stroke survivors can learn to control the wrist flexion almost without activating the antagonist muscles. Despite the encouraging results we found a significant increase of antagonists' activity in the 3rd week. A possible explanation can be a variable level of spasticity, attention or daily fatigue [24] that are very common in wrist muscles (m. carpi ulnaris/radialis). In addition to the desired muscle activity we may have also assessed the daily increased spastic activities of other muscle fibers with the surface HDEMG. The presence of muscle fatigue and insufficient residual muscle activity can often present a problem and a major disadvantage of EMG interfaces in rehabilitation [25]. Additionally the failure of a single electrode can provide a misleading EMG biofeedback. However, the proposed approach with HDEMG can overcome and eliminate the misleading electromyographic information from neighboring muscle activities. For example the detailed analysis of raw HDEMG also shows that in spite of the significantly higher RMS in the $3^{\text {rd }}$ week we have not noticed a clear co-activation similar to the one of the antagonists at wrist extension but rather a constant muscle activity. For a successful selection of desired motor units [9] we may further examine the mesh of RMS HDEMG values. 
The HDEMG with 64 + electrodes may be convenient for automatic and real-time information processing in neural recording for brain computer interfaces [26], but presented a challenge for biofeedback.

Therefore we shrank the information to only 4 regions for each muscle group and demonstrated the feasibility of the approach in a single person after stroke. The participant was able to follow the biofeedback, keeping the target force/muscle activity within desired limits and activating predominantly the agonist muscle group. However, the concentration and motivation may play an important role in the biofeedback, the visualization of muscle activity [27] in particular. The presented approach can increase the reliability and precision of existing successful applications of EMG biofeedback [11]. Therefore we should proceed further with a study in a larger group.

\section{Limitations And Strengths}

The major limitation of all and also this feasibility study is that it can not provide a proof of clinical or any other effectiveness of the presented approach, but rather demonstrates the applicability of the device and feasibility of the novel methods used. Therefore the presented method should be explored in a larger group of stroke survivals and its effectiveness confirmed by a randomized control study.

But the strength of the presented novelty is the high-density surface electromyography with information reduction on the basis of computational filtering [17] in real-time, making it suitable for the biofeedback in wrist rehabilitation. In fact the surface HDEMG can ensure solidity, add redundancy and successfully eliminates movement artifacts resulting in the improved decoding reliability [28].

\section{Conclusion}

The UHD robot ensured isolated wrist flexion and extension and we have demonstrated that the surface HDEMG biofeedback has a potential to decrease the co-activation of antagonist muscles, for wrist flexion in particular. Additionally the combination of force tracking and biofeedback training may recover selective hand function in stroke survivors who would less likely to develop contractures. However, a clinical study in a large group of stroke survivors is essential to provide an evidence.

\section{Declarations}

\section{Ethics approval and consent to participate}

The study (Approval Number: URI201728) was approved by ethics committee of University Rehabilitation Institute, Republic of Slovenia and all participants provided an informed written consent. The procedure was in accordance with the principles of the Declaration of Helsinki on biomedical research on human beings, the provisions of Council of Europe Convention on the Protection of Human Rights and Dignity of the Human Being with regard to the Application of Biology and Medicine (Oviedo Convention) and the principles of Slovenian Code of medical ethics. 


\section{Consent for publication}

All authors, project group members and participants agreed on publication. Participants provided a written consent for the publication of photographs.

\section{Availability of data and material}

Anonymized data generated or analyzed during this study are included in this published article [and its supplementary information files]

\section{Conflict of Interest}

None declared.

\section{Funding}

Slovenian Research Agency (research core funding No. P2-0228)

\section{Author contributions}

IC, MZ, FU, MD, AF, JK carried out the measurements. MŠ, JK, BP performed the signal and data processing, IC performed the data and statistical analysis, participated in the sequence alignment and drafted the manuscript. AH and ZM helped to draft the manuscript. AH conceived the study and with ZM, IC and MD participated in its design and coordination. All authors read and approved the final manuscript.

\section{Acknowledgements}

The project was funded by the Slovenian Research Agency (research core funding No. P2-0228, P2-0041 and project funding ID J2-7357).

\section{References}

1. Theories Of Learning. Ernest R. Hilgard : Free Download, Borrow, and Streaming : Internet Archive [Internet]. [cited 2020 May 13]. Available from: https://archive.org/details/TheoriesOfLearning/mode/2up.

2. Huang $\mathrm{H}$, Wolf $\mathrm{SL}$, He J. Recent developments in biofeedback for neuromotor rehabilitation. J Neuroeng Rehabil [Internet]. BioMed Central; 2006 [cited 2020 Apr 6];3:11. Available from: http://www.ncbi.nlm.nih.gov/pubmed/16790060.

3. $10.1186 / 1743-0003-10-60$ 
Giggins OM, Persson UMC, Caulfield B. Biofeedback in rehabilitation [Internet]. J. Neuroeng. Rehabil. BioMed Central; 2013 [cited 2020 May 7]. p. 60. Available from: http://jneuroengrehab.biomedcentral.com/articles/10.1186/1743-0003-10-60.

4. Armagan O, Tascioglu F, Oner C. Electromyographic Biofeedback in the Treatment of the Hemiplegic Hand. Am J Phys Med Rehabil [Internet]. 2003 [cited 2020 Apr 6];82:856-61. Available from: http://journals.Iww.com/00002060-200311000-00006.

5. Kim JH. The effects of training using EMG biofeedback on stroke patients upper extremity functions. J Phys Ther Sci Society of Physical Therapy Science (Rigaku Ryoho Kagakugakkai). 2017;29:10858.

6. Belén Gámez A, José Hernandez Morante J, Luis J, Gil M, Esparza F, Martínez CM. The effect of surface electromyography biofeedback on the activity of extensor and dorsiflexor muscles in elderly adults: a randomized trial. [cited 2020 Apr 6]; Available from: www.nature.com/scientificreports.

7. Rojas-Martínez M, Mañanas MA, Alonso JF. High-density surface EMG maps from upper-arm and forearm muscles. J Neuroeng Rehabil BioMed Central. 2012;9:85.

8. Drost G, Stegeman DF, van Engelen BGM, Zwarts MJ. Clinical applications of high-density surface EMG: A systematic review. J. Electromyogr. Kinesiol. Elsevier; 2006. p. 586-602.

9. Glaser V, Holobar A. Motor unit identification from high-density surface electromyograms in repeated dynamic muscle contractions. IEEE Trans Neural Syst Rehabil Eng Institute of Electrical Electronics Engineers Inc. 2019;27:66-75.

10. Geethanjali P. Myoelectric control of prosthetic hands: State-of-the-art review. Med. Devices Evid. Res. Dove Medical Press Ltd; 2016. p. 247-55.

11. Rayegani S, Raeissadat S, Sedighipour L, Rezazadeh I, Bahrami M, Eliaspour D, et al. Effect of neurofeedback and electromyographic-biofeedback therapy on improving hand function in stroke patients. Top Stroke Rehabil. Thomas Land Publishers Inc.; 2014;21:137-51.

12. Davis AE, Lee RG. EMG Biofeedback in Patients with Motor Disorders: An Aid for Co-Ordinating Activity in Antagonistic Muscle Groups. Can J Neurol Sci / J Can des Sci Neurol. 1980;7:199-206.

13. Mugler EM, Tomic G, Singh A, Hameed S, Lindberg EW, Gaide J, et al. Myoelectric Computer Interface Training for Reducing Co-Activation and Enhancing Arm Movement in Chronic Stroke Survivors: A Randomized Trial. Neurorehabil Neural Repair. SAGE Publications Inc.; 2019;33:284-95.

14. Oblak J, Cikajlo I, Matjaĉić Z. Universal haptic drive: A robot for arm and wrist rehabilitation. IEEE Trans Neural Syst Rehabil Eng. 2010.

15. Perry JC, Oblak J, Jung JH, Cikajlo I, Veneman JF, Goljar N, et al. A variable structure pantograph mechanism for comprehensive upper extremity haptic movement training. J Rehabil Res Dev [Internet]. 2010;48:317-33. Available from: http://www.ncbi.nlm.nih.gov/pubmed/21096924.

16. Journal of Statistics Education, V11N1. Hays [Internet]. [cited 2020 Apr 23]. Available from: http://jse.amstat.org/v11n1/datasets.hays.html.

17. Potocnik B, Holobar A, Divjak M, Urh F, Francic A, Kranjec J, et al. Estimation of muscle co-activations in wrist rehabilitation after stroke is sensitive to motor unit distribution and action potential shapes. 
IEEE Trans Neural Syst Rehabil Eng. Institute of Electrical and Electronics Engineers (IEEE); 2020;1-1.

18. Savc M, Glaser V, Kranjec J, Cikajlo I, Matjacic Z, Holobar A. Comparison of Convolutive Kernel Compensation and Non-Negative Matrix Factorization of Surface Electromyograms. IEEE Trans Neural Syst Rehabil Eng [Internet]. 2018 [cited 2018 Nov 8];26:1935-44. Available from: http://www.ncbi.nlm.nih.gov/pubmed/30281464.

19. Standards for Performing Biofeedback - AAPB [Internet]. [cited 2020 May 7]. Available from: https://www.aapb.org/i4a/pages/index.cfm?pageid=3678.

20. De Luca CJ, Donald Gilmore L, Kuznetsov M, Roy SH. Filtering the surface EMG signal: Movement artifact and baseline noise contamination. J Biomech Elsevier. 2010;43:1573-9.

21. Kurillo G, Zupan A, Bajd T. Force tracking system for the assessment of grip force control in patients with neuromuscular diseases. Clin Biomech Elsevier. 2004;19:1014-21.

22. Chang E, Ghosh N, Yanni D, Lee S, Alexandru D, Mozaffar T. A review of spasticity treatments: Pharmacological and interventional approaches. Crit Rev Phys Rehabil Med NIH Public Access. 2013;25:11-22.

23. Malhotra S, Pandyan AD, Rosewilliam S, Roffe C, Hermens H. Spasticity and contractures at the wrist after stroke: Time course of development and their association with functional recovery of the upper limb. Clin Rehabil. 2011;25:184-91.

24. Li S. Spasticity, motor recovery, and neural plasticity after stroke. Front. Neurol. Frontiers Research Foundation; 2017. p. 120.

25. Kastalskiy I, Mironov V, Lobov S, Krilova N, Pimashkin A, Kazantsev V. A Neuromuscular Interface for Robotic Devices Control. Comput Math Methods Med. 2018;2018.

26. Kosmyna N, Maes P. AttentivU: An EEG-Based Closed-Loop Biofeedback System for Real-Time Monitoring and Improvement of Engagement for Personalized Learning. Sensors [Internet]. MDPI AG; 2019 [cited 2020 May 7];19:5200. Available from: https://www.mdpi.com/1424-8220/19/23/5200.

27. Sturma A, Hruby LA, Prahm C, Mayer JA, Aszmann OC. Rehabilitation of upper extremity nerve injuries using surface EMG biofeedback: Protocols for clinical application. Front Neurosci [Internet]. Frontiers Media S.A.; 2018 [cited 2020 May 11];12:906. Available from: http://www.ncbi.nlm.nih.gov/pubmed/30564090.

28. Martinez-Valdes E, Laine CM, Falla D, Mayer F, Farina D. High-density surface electromyography provides reliable estimates of motor unit behavior. Clin Neurophysiol Elsevier Ireland Ltd. 2016;127:2534-41.

\section{Figures}


EXTENSORS ELECTRODE $5 \times 13$

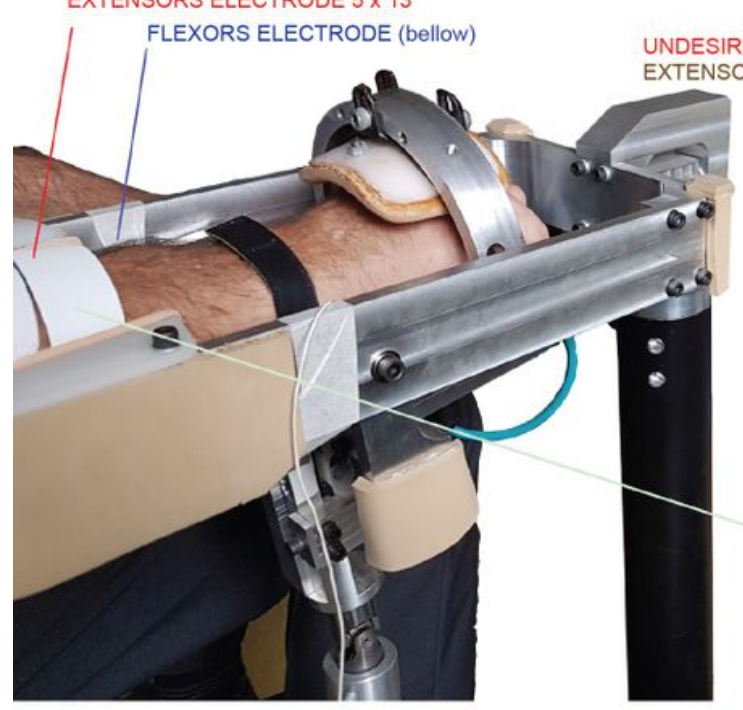
MUSCLES

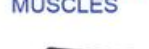
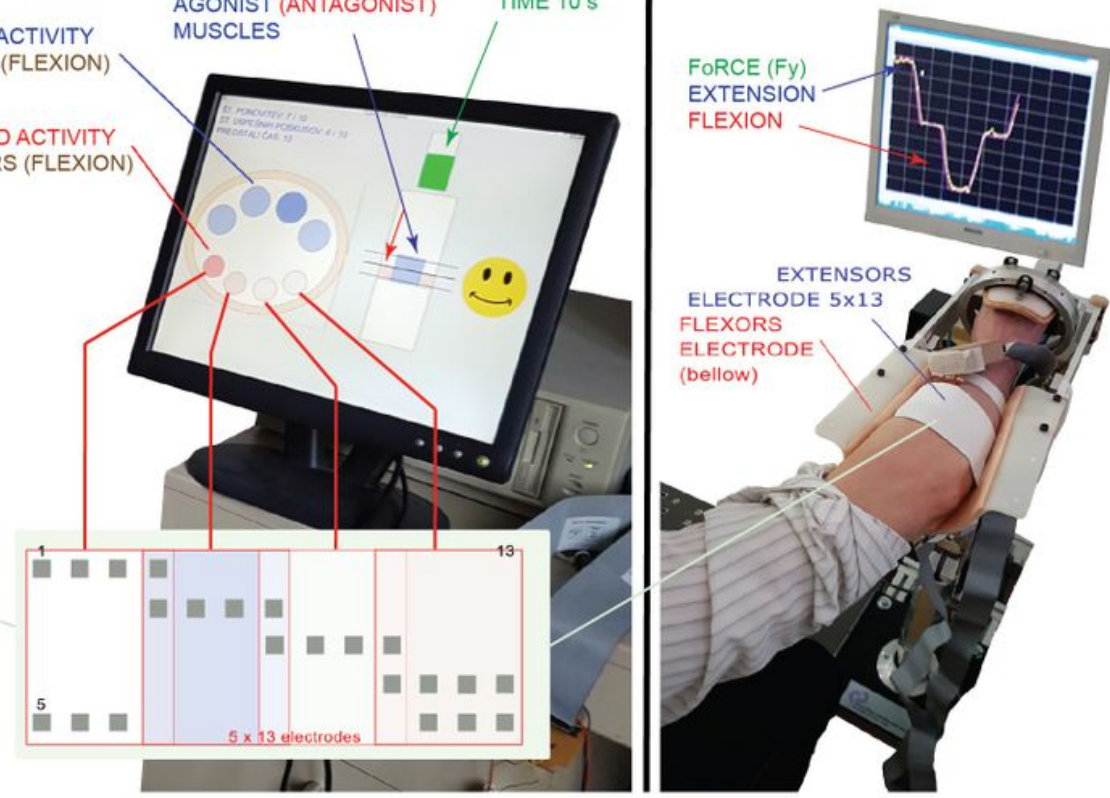

(bellow)

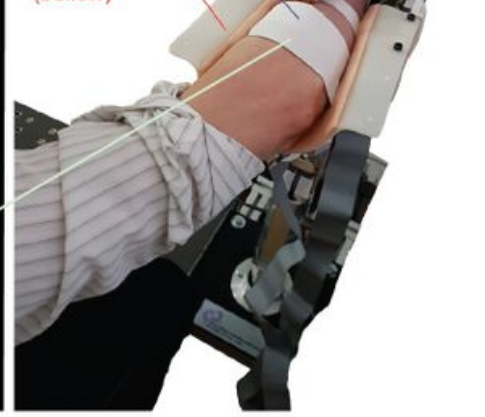

Figure 1

HDEMG robot biofeedback system for wrist rehabilitation. Left: keeping the agonist muscles activity and reducing the antagonist muscles activity in appropriate flexion/extension for $10 \mathrm{~s}$. The cumulative agonist muscles activity for 10 s (green bar) is considered a successful trial and is granted a smiley. Right: force tracking by flexion/extension presented on the display. 


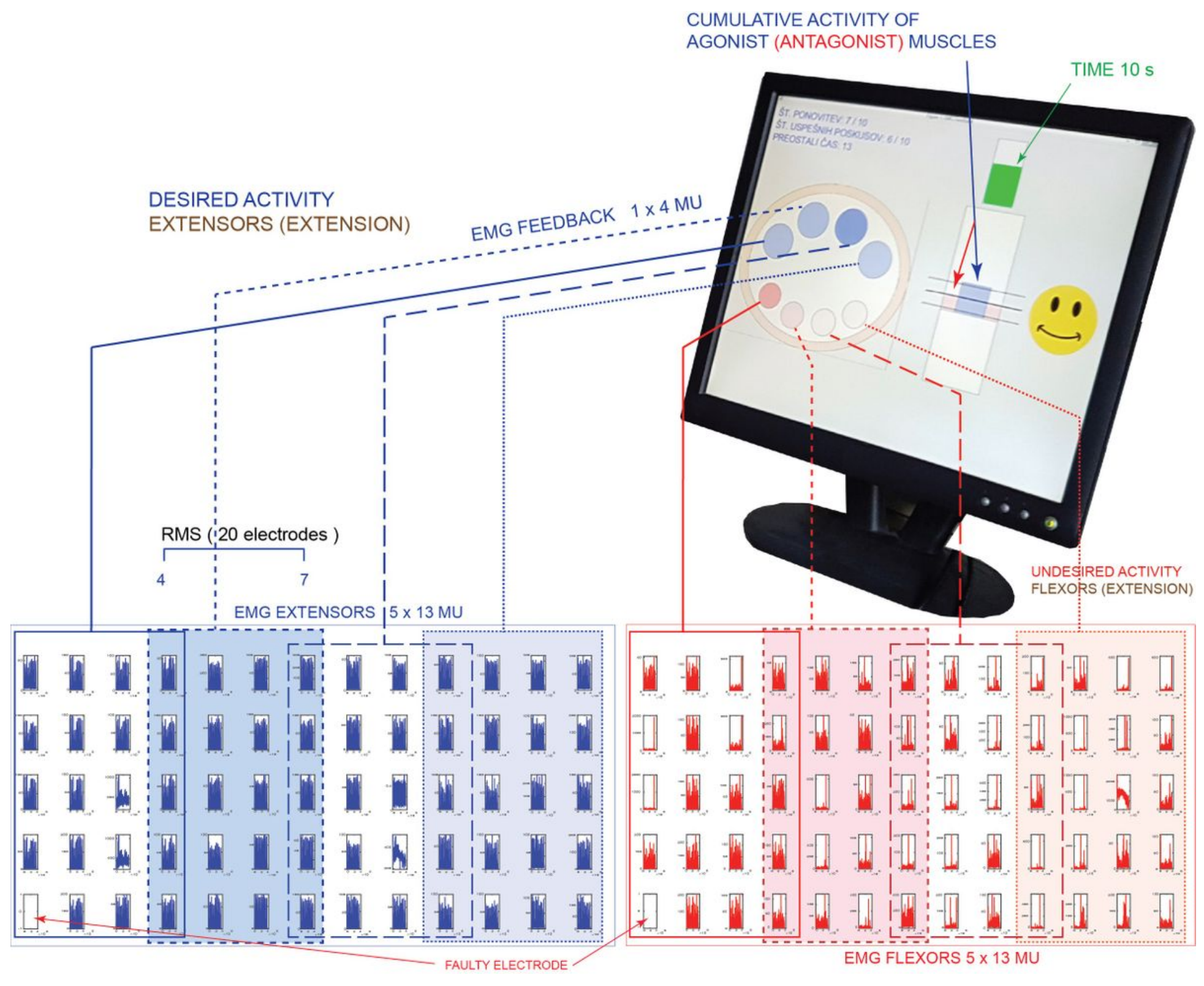

Figure 2

The signals of faulted electrodes were eliminated from the feedback and analysis. The major challenge was decreasing the data from $5 \times 13$ multi-array electrodes to the reliable muscle contraction information for biofeedback in $1 \times 4$ format. The cumulative antagonists activity (red) pushes the target line higher for the required cumulative agonistic muscles activity. Keeping up with the target for $10 \mathrm{~s}$ (green bar) gets a smiley. 

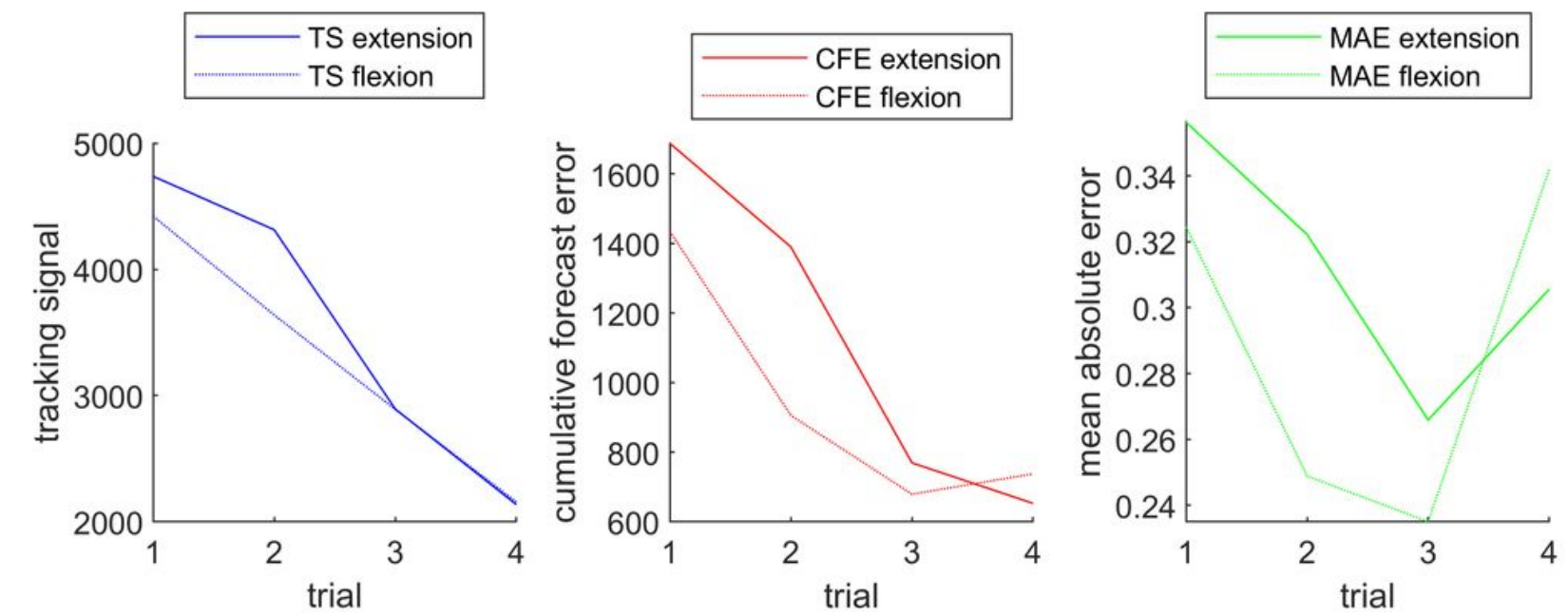

\section{Figure 3}

The tracking of the reference force required alternating wrist flexion and extension. The UHD robot inhibited the movement by providing linearly opposite force to movement. The participant successfully managed to keep up with the reference successfully.

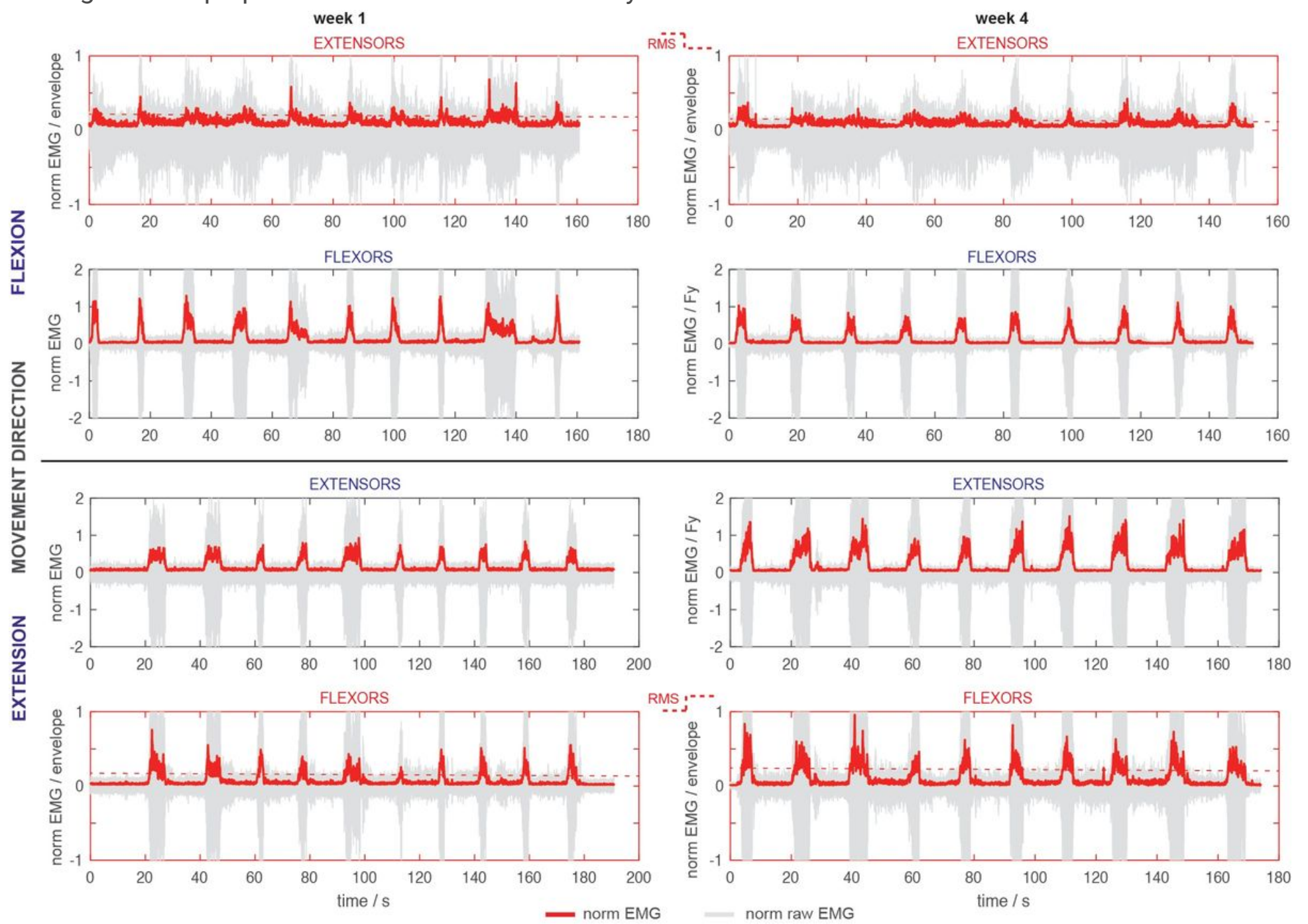


Figure 4

The normalized and rectified EMG demonstrated reduced activity of antagonists (extensor) muscles during wrist flexion exercise with biofeedback after 4 weeks. The raw EMG also showed reduced but continuous muscle contractions. However, the co-contraction of antagonists (flexors) with extensors during wrist extension was even a bit higher after 4 weeks than during the first week.

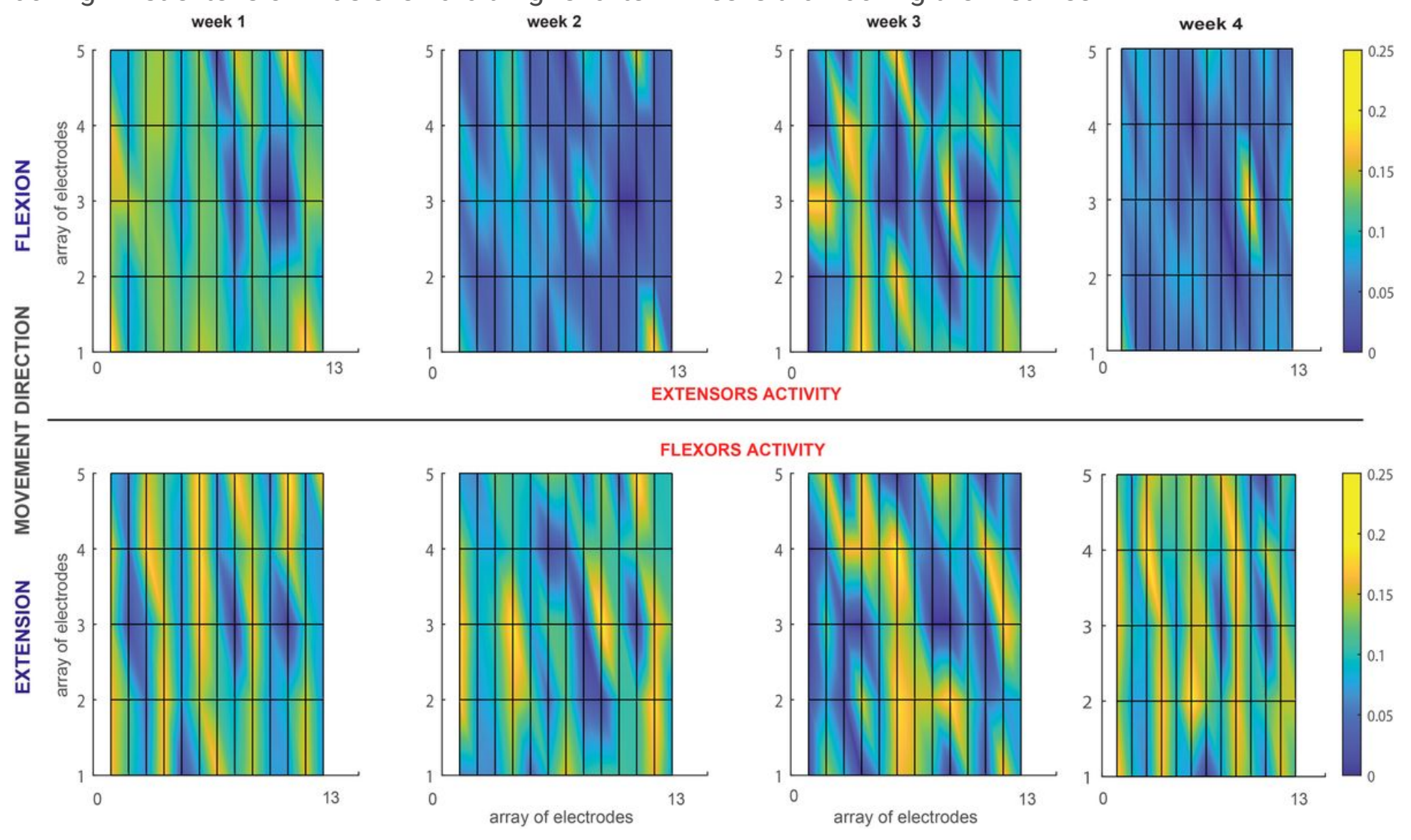

Figure 5

The mesh of RMS of HDEMG shows gradually less activity (blue) of antagonistic muscles at wrist flexion over the 4 weeks, but also slightly and in places higher activity (yellow) of antagonist at wrist extension. 

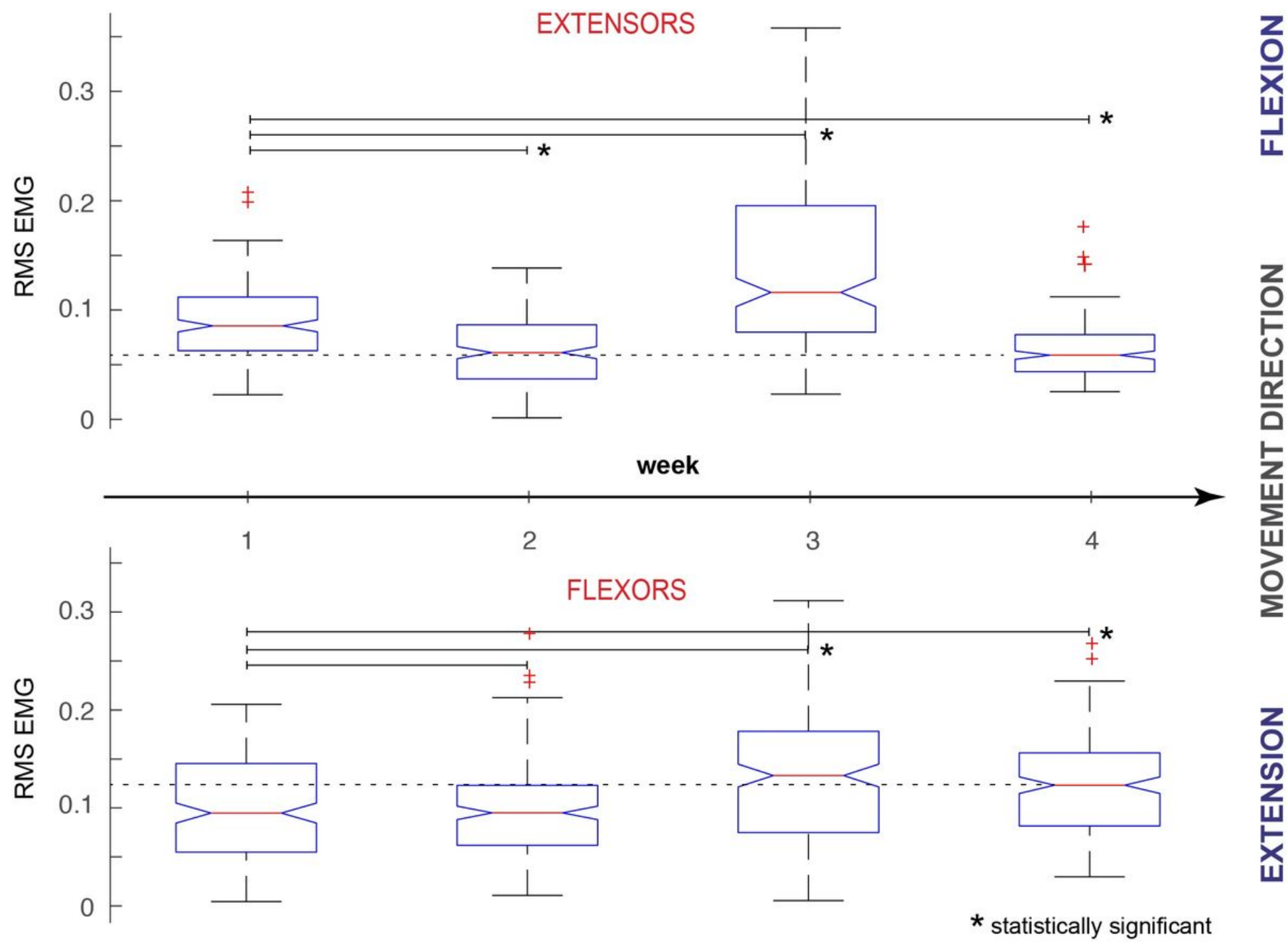

Figure 6

Statistical analysis of RMS of HDEMG over the 4 weeks. Kruskal-Wallis test was used to confirm the significantly different outcomes between the 1 st and the following weeks. The important finding was the decrease of antagonists' contraction during wrist flexion; may be due to a lower spasticity, but further research in a larger group of participants is needed to confirm these findings. Interquartile range (IQR),1.5 times IQR, 95\% confidence intervals and outliers (+) are presented. 